\title{
Apelin promotes Iymphangiogenesis and lymph node metastasis
}

\author{
Judit Berta ${ }^{1,2, *}$, Mir Alireza Hoda ${ }^{1, *}$, Viktoria Laszlo ${ }^{1,3}$, Anita Rozsas ${ }^{1,2}$, Tamas \\ Garay $^{2,3,4}$, Szilvia Torok ${ }^{2}$, Michael Grusch ${ }^{5}$, Walter Berger ${ }^{5}$ Sandor Paku ${ }^{3,6}$, \\ Ferenc Renyi-Vamos ${ }^{1,7}$, Bernard Masri, ${ }^{8}$ Jozsef Tovari ${ }^{9}$, Marion Groger ${ }^{10,11}$, Walter \\ Klepetko $^{1}$, Balazs Hegedus ${ }^{1,4, * *}$, Balazs Dome ${ }^{1,2,7, * *}$ \\ ${ }^{1}$ Division of Thoracic Surgery, Department of Surgery, Comprehensive Cancer Center, Medical University of Vienna, Austria \\ 2 Department of Tumor Biology, National Koranyi Institute of Pulmonology, Budapest, Hungary \\ ${ }^{3}$ Department of Biological Physics, Eötvös Loránd University, Budapest, Hungary \\ ${ }^{4}$ MTA-SE Molecular Oncology Research Group, Hungarian Academy of Sciences, Budapest, Hungary \\ ${ }^{5}$ Institute of Cancer Research and Comprehensive Cancer Center, Department of Medicine I, Medical University of Vienna, \\ Austria \\ ${ }^{6}$ 1st Department of Pathology and Experimental Cancer Research, Semmelweis University, Budapest, Hungary \\ 7 Thoracic Surgery, National Institute of Oncology and Semmelweis University, Budapest, Hungary \\ ${ }^{8}$ Cancer Research Center of Toulouse, INSERM U1037-Université Paul Sabatier Toulouse III, Toulouse, France \\ 9 Department of Experimental Pharmacology, National Institute of Oncology, Budapest, Hungary; Skin and Endothelium \\ Research Division (SERD) \\ 10 Department of Dermatology, Medical University of Vienna, Austria \\ ${ }^{11}$ Core Facility Imaging, Core Facilities, Medical University of Vienna, Austria \\ * These Authors contributed equally to this work \\ ** These authors share last authorship \\ Correspondence to: Balazs Dome, email: balazs.dome@meduniwien.ac.at \\ Keywords: apelin, APJ, lymphangiogenesis, lymph node metastasis \\ Received: April 8, $2014 \quad$ Accepted: May 27, $2014 \quad$ Published: May 28, 2014
}

This is an open-access article distributed under the terms of the Creative Commons Attribution License, which permits unrestricted use, distribution, and reproduction in any medium, provided the original author and source are credited.

\section{ABSTRACT}

Whereas the role of the G-protein-coupled APJ receptor and its ligand, apelin, in angiogenesis has been well documented, the ability of the apelin/APJ system to induce lymphangiogenesis and lymphatic metastasis has been largely unexplored. To this end, we first show that APJ is expressed in lymphatic endothelial cells (LECs) and, moreover, that it responds to apelin by activating the apelinergic signaling cascade. We find that although apelin treatment does not influence the proliferation of LECs in vitro, it enhances their migration, protects them against UV irradiationinduced apoptosis, increases their spheroid numbers in 3D culture, stimulates their in vitro capillary-like tube formation and, furthermore, promotes the invasive growth of lymphatic microvessels in vivo in the matrigel plug assay. We also demonstrate that apelin overexpression in malignant cells is associated with accelerated in vivo tumor growth and with increased intratumoral lymphangiogenesis and lymph node metastasis. These results indicate that apelin induces lymphangiogenesis and, accordingly, plays an important role in lymphatic tumor progression. Our study does not only reveal apelin as a novel lymphangiogenic factor but might also open the door for the development of novel anticancer therapies targeting lymphangiogenesis.

\section{INTRODUCTION}

The lymphatic system is essential for maintaining interstitial fluid homeostasis, immune cell trafficking and lipid transport. The lymphatic network also has an active role in different pathological conditions including tissue 
inflammation, wound healing, renal and corneal graft rejection [1] but lymphatics are insufficient in patients with primary or secondary lymphoedema [2]. Although lymphatic spread frequently occurs in solid malignancies and is considered an indicator of local dissemination and poor prognosis, a long-established concept has assigned a passive role to lymphatics in tumor progression. However, recent animal studies using lymphangiogenic molecules have indicated that lymphatic vessels interact extensively with malignant cells and, moreover, that lymphangiogenesis is associated with, and can enhance, lymph node (LN) metastasis. The most widely studied molecular system that facilitates the expansion of the lymphatic network both under physiological and pathological conditions is the VEGF-C/ -D / VEGFR-3 signaling pathway. Nevertheless, our knowledge of the molecular mechanisms that underlie lymphangiogenesis still lags far behind that of the vascular system [3].

Apelin has been recognized as the endogenous ligand of the human G protein-coupled receptor APJ [4], a member of the seven-transmembrane-receptor family. During embryonic development, APJ expression is largely restricted to the endothelial cells of the developing vascular system [5] and apelin is essential for vascular patterning of the embryo [6]. Nevertheless, apelin and its receptor are strongly expressed in the adult blood vasculature as well [7], and apelin was reported to stimulate blood endothelial cell growth in various in vitro $[8,9]$ and in vivo $[6,9]$ angiogenesis models. It has also been demonstrated that apelin can induce the maturation of tumor blood capillaries [10] and, moreover, that the apelin-APJ system is able to increase the vascularization and growth of different murine tumors [11]. Moreover, apelin was found to be upregulated in some human cancers [12-14], and both our group [15] and others [16, 17] demonstrated a direct association of apelin expression with angiogenesis and/or clinical outcome in malignant disease.

Apelin probably also has lymphangiogenic potential, however, evidence supporting this view has hitherto been obtained only in two very recent studies on the role of apelin in inflammation [18] and lymphatic development [19]. Therefore, and given the apelinergic system's well established angiogenic potential under both physiological and pathological conditions, the present study was conducted to examine additional aspects of the apelin-APJ pathway in lymph vessel growth.

Here, we use in vitro and in vivo assays to dissect the role of apelin in lymphangiogenesis and lymphatic tumor spread. First, we investigate APJ expressions of LECs and analyze the downstream pathways upon activation of APJ signaling. Next, we assess LECs' in vitro proliferation, migration, apoptosis and tube as well as spheroid formation following apelin treatment. We also quantify the in vivo effect of exogenous apelin on lymph vessel growth in the Matrigel plug system. Finally, we study whether transfection of tumor cells with apelin expression constructs results in an increase in lymphangiogenesis and LN metastasis in vivo. Our results provide the first direct evidence that apelin induces lymphangiogenesis and lymphatic metastasis.

\section{RESULTS}

\section{APJ is expressed by human LECs and apelin activates its downstream signaling}

RT-PCR and immunocytochemical analysis of HUVECs (human umbilical vein endothelial cells) and LECs demonstrated the presence of APJ mRNA (Fig. 1.A) and protein (Fig. 1. B-C) expressions, respectively. In order to demonstrate that APJ is functionally active in LECs, we investigated the activation of previously described downstream signaling molecules $[8,20]$. We found that apelin led to a significantly increased phosphorylation of Erk1/2 and Akt after 5 and 15 minutes treatment, respectively. Activation of S6 was not significant following apelin treatment (Fig. 1. D-E). Thus, APJ expressed in human LECs responds to apelin treatment by inducing the activation of the Erk and PI3KAkt pathways as we described in other cell types as well $[8,20]$.

\section{Apelin does not increase the in vitro growth of LECs but promotes their migration and protects them against apoptosis}

To investigate whether apelin alters LEC growth in vitro, the effect of apelin treatment on cell proliferation was studied by BrdU incorporation assay. Of note, exogenous apelin did not alter the in vitro proliferation rate of these cells, when compared with untreated cells after $96 \mathrm{~h}$ (Fig. 2. A-B).

Apelin has been shown to reduce apoptosis in various cell types, including retinal Müller cells [21], osteoblasts [22], neurons [23], vascular smooth muscle cells [24] and blood endothelial cells as well [6]. We used TUNEL analysis to investigate whether the apelin-APJ system can also inhibit programmed cell death in LECs. Using a standard serum starvation protocol, we found that $48 \mathrm{~h}$ serum deprivation did not result in significant apoptosis induction in LECs. However, administration of 1 $\mu \mathrm{M}$ apelin significantly suppressed $\mathrm{UV}$ irradiation-induced apoptosis of LECs $(\mathrm{P}<0.05$; Fig. 2. C-F).

Next, we sought to determine the effect of apelin on human LEC migration. Using long-term time-lapse videomicroscopy in 2D cell cultures [25] (Fig. 3. A-B), we found that apelin at $100 \mathrm{nM}$ significantly increased cell migration $(\mathrm{P}<0.05$; Fig. 3. C-D), indicating a pro- 

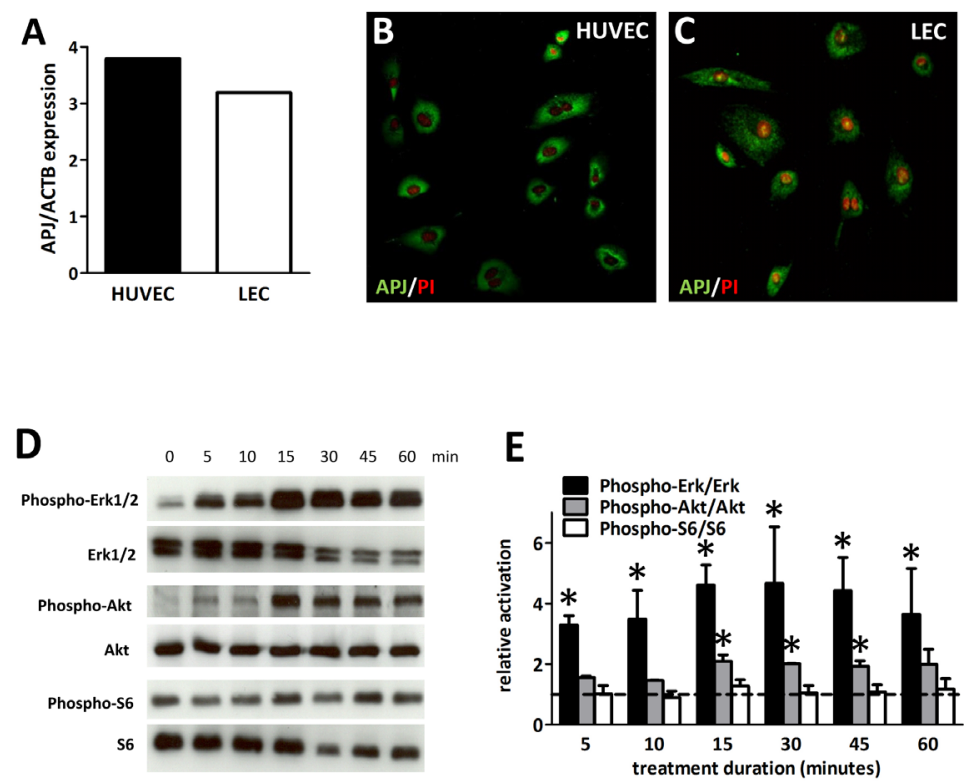

Figure 1: Expression and activation of APJ receptor in LECs. (A) In LECs, APJ mRNA was detected at a comparable level to that in HUVECs. (B), Immunofluorescent staining of APJ (green) in HUVECs and LECs. Nuclei were labeled with PI (red). (D), Time course of phosphorylation of Erk1/2, Akt and S6 following $1 \mu \mathrm{M}$ apelin-13 treatment of LECs. (E), Quantification of activation by the ratio of the phosphorylated and total protein levels shows robust and significant activation of Erk $1 / 2$ and Akt. No significant activation of $\mathrm{S} 6$ was found upon apelin treatment. Asterisks designate significant differences $(\mathrm{P}<0.05)$. Columns represent mean of three experiments; bars, SEM.
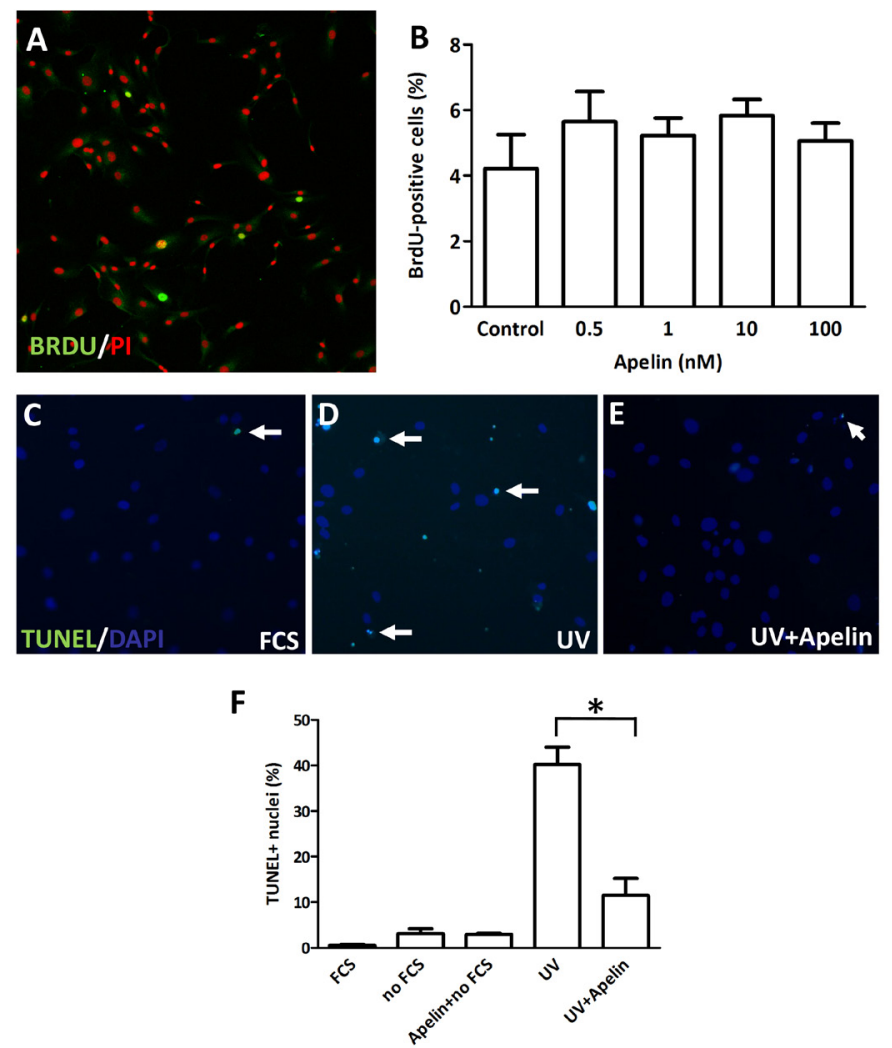

Figure 2: Proliferation and apoptosis of LECs following apelin treatment. (A), LECs were cultured in serum-free medium and treated with apelin. After 96 hours, cells were pulsed with BrdU, fixed and stained with an anti-BrdU antibody (green) and PI (red). (B), No significant change was found in the ratio of BrdU-labeled nuclei upon apelin treatments. (C-E), Apoptosis induction with UV in LECs. Apoptotic cells are labeled with TUNEL (green). DAPI nuclei staining is blue. White arrows indicate apoptotic nuclei. (F), Robust apoptosis was induced by UV irradiation. Apelin treatment significantly reduced the ratio of UV induced apoptotic cells. Asterisks designate significant differences $(\mathrm{P}<0.05)$. In $(\mathrm{B})$ and $(\mathrm{F})$, columns represent mean of three experiments; bars, SD. 
migratory potential for apelin in addition to its antiapoptotic role in human LECs. Importantly, while APJ inhibition by F13A (an APJ antagonist) alone did not result in significant changes in cell migration, it reduced apelininduced LEC migration in a dose-dependent manner with complete inhibition when apelin and F13A was given at 1:1 ratio $(\mathrm{P}<0.05$; Fig. 3. C-D). Representative pictures of the LECs' trajectories and their average migrated distance for $12 \mathrm{~h}$ intervals are also shown in Figure 3. A-B.

\section{Apelin increases LEC spheroid numbers and stimulates capillary-like cord formation of LECs in vitro and, moreover, promotes the growth of lymph vessels in the Matrigel plug model in vivo}

Three-dimensional spheroid endothelial cell models (introduced by Korff and Augustin in 1998 [26]) are useful tools for the analysis of the activity of pro- and antiangiogenic factors. Because apelin-APJ signaling has been reported to enhance proliferation and assembly of blood endothelial cells in spheroid cultures [27], we also tested the effects of apelin on the formation of multicellular spheroid structures by LECs (Fig. 4). Although apelin treatments at different doses $(10 \mathrm{nM}$ or $1 \mu \mathrm{M})$ did not result in significantly elevated mean spheroid diameters
(Fig. 4. C), apelin (at $1 \mu \mathrm{M}$ ) significantly increased the number of LEC spheroids, when compared with untreated cells after $96 \mathrm{~h}(\mathrm{P}<0.05$; Fig. 4. D).

Endothelial tube formation assays are also regarded as in vitro models of more advanced stages of (lymph) angiogenesis and are also often used to test the effects of different pro- and anti-(lymph)angiogenic molecules [28]. Thus, we examined the ability of apelin to promote LEC tube formation in a Matrigel tube formation assay and found that apelin (at $1 \mu \mathrm{M}$ ) was effective in promoting LEC differentiation into vascular structures in vitro $(\mathrm{P}<0.05$ vs. control; Fig. 5. D). The extent of this effect was comparable to that of bFGF (basic fibroblast growth factor) at $20 \mathrm{ng} / \mathrm{mL}$, which was used as a positive control $(\mathrm{P}<0.05$ vs. control; Fig. 5. D).

The Matrigel plug technique was used to study the effect of apelin on the invasive growth of lymphatic capillaries in mice. Corroborating the lymphangiogenic potential of apelin seen in the above described in vitro assays, LVDs were significantly higher in Matrigel plugs containing apelin $(0.25 \mu \mathrm{g})$ or bFGF $(0.25 \mu \mathrm{g})$ than in those with PBS only $(\mathrm{P}<0.05$ in case of both apelin and bFGF); Fig. 5. E).

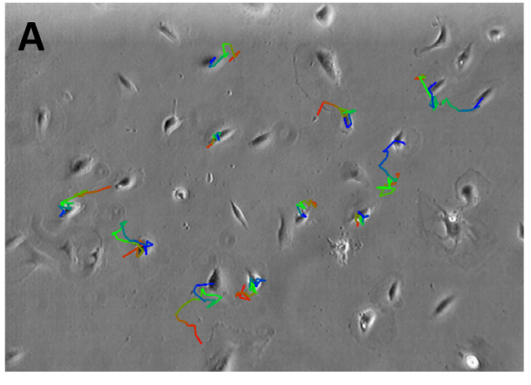

C

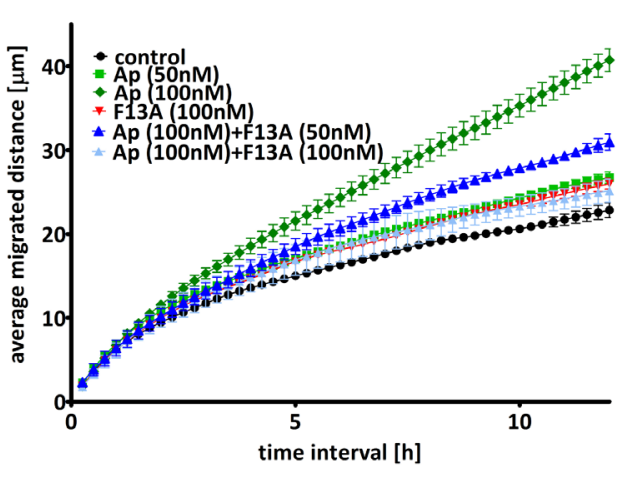

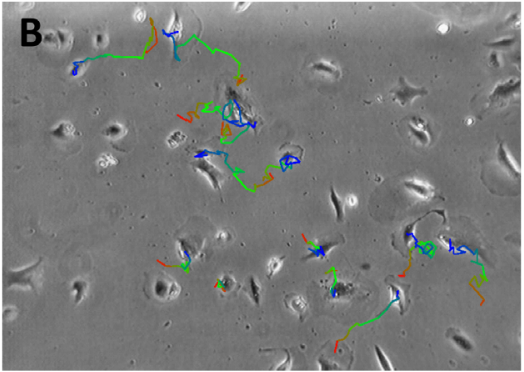

D

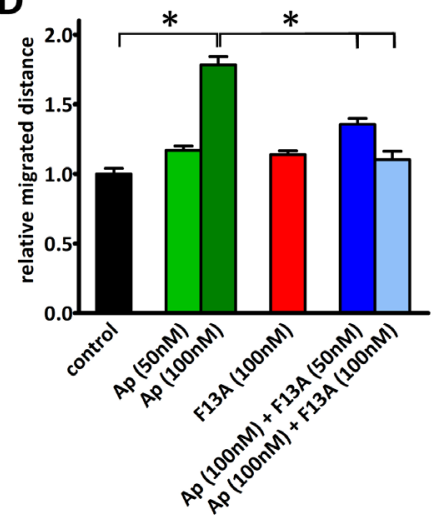

Figure 3: Effect of apelin on LEC migration. (A-B), Representative trajectories on the last phase contrast image of the path of LECs with (B) or without (A) apelin treatment. The color of the depicted trajectories refers to the elapsed time in the order from red-greenblue. (C), Average migrated distances of human LECs after incubation with apelin-13 and/or F13A (an APJ antagonist). (D), 12h average migrated distance of LECs after incubation with $100 \mathrm{nM}$ apelin-13 significantly increased cell migration and it was reduced in a dose dependent manner by the addition of F13A. Asterisks designate significant differences $(\mathrm{P}<0.05)$. 
Apelin overexpression confers a growth advantage to tumor grafts, induces intratumoral lymphangiogenesis and promotes lymphatic metastasis
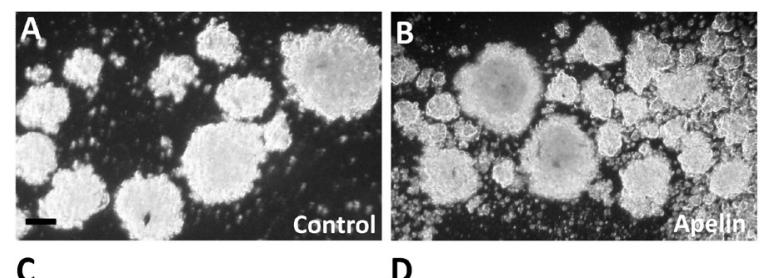

D
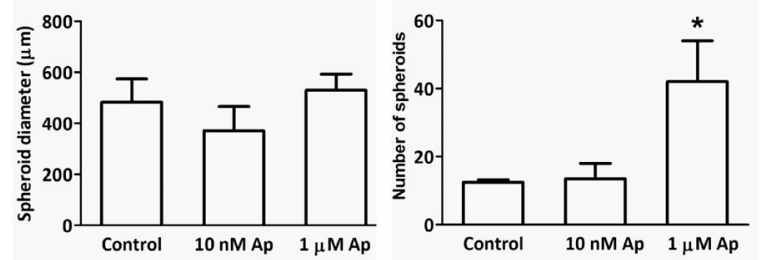

Figure 4: LEC spheroid formation in the presence of apelin. (A-B), LECs were plated in serum-free medium in non-adherent plates with apelin-13. After 96h incubation, all spheroids were photographed. (C-D), $1 \mu \mathrm{M}$ apelin-13 significantly increased the number of spheres without affecting their average diameter. Columns represent mean of three experiments; bars, $\mathrm{SD} ; *, \mathrm{P}<0.05$ vs. control. Scale bar $100 \mu \mathrm{m}$

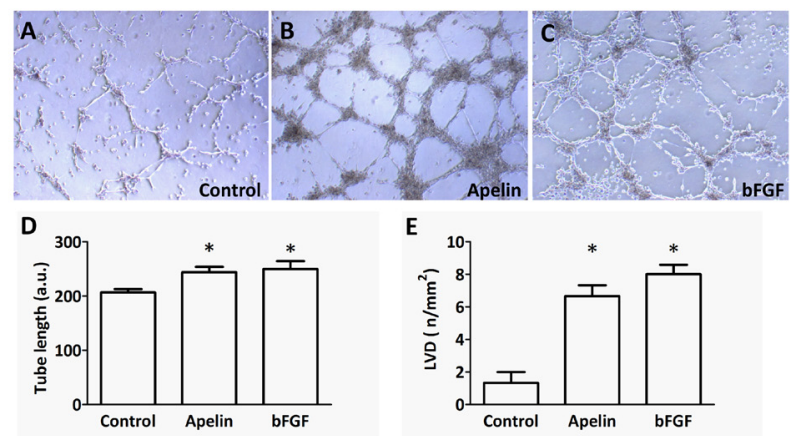

Figure 5: Tube formation capacity of LECs upon apelin treatment in vitro and in vivo. (A-C), LECs $\left(10^{5}\right)$ were plated on growth factor-reduced Matrigel-coated tissue culture plates in serum-free endothelial culture medium. $1 \mu \mathrm{M}$ apelin- 13 or $20 \mathrm{ng} / \mathrm{ml} \mathrm{bFGF}$ was added to the medium. Capillarylike structures within the Matrigel layer were photographed after 18 hours. (D), Both apelin and bFGF significantly increased the average length of tubes $(*, P<0.05$ vs. control). Columns represent mean of three experiments; bars, SD; a.u., arbitrary unit (E), Matrigel containing PBS, apelin-13 $(0.25 \mu \mathrm{g})$ or bFGF $(0.25 \mu \mathrm{g})$ was injected subcutanously into mice. Both bFGF and apelin-13 significantly increased the density of LYVE-1 stained tubular structures in the sections of one-week old plugs. Columns represent means of three experiments; bars, SD; *, $\mathrm{P}<0.05$ vs. control. LVDs are mean lymph vessel counts per square millimeter.
We were also interested in investigating whether transfection of tumor cells with apelin expression constructs results in an increase in tumor lymphangiogenesis in vivo. Our studies to address this goal involved experiments with B16 mouse melanoma cells stably transfected with murine apelin (B16-apelin)
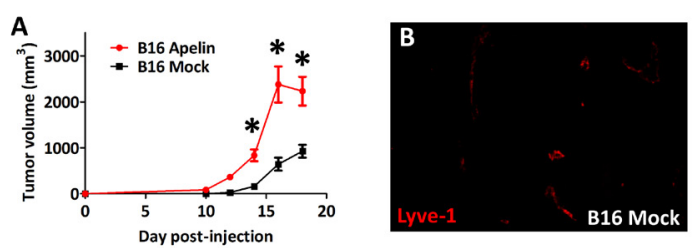

\section{C}
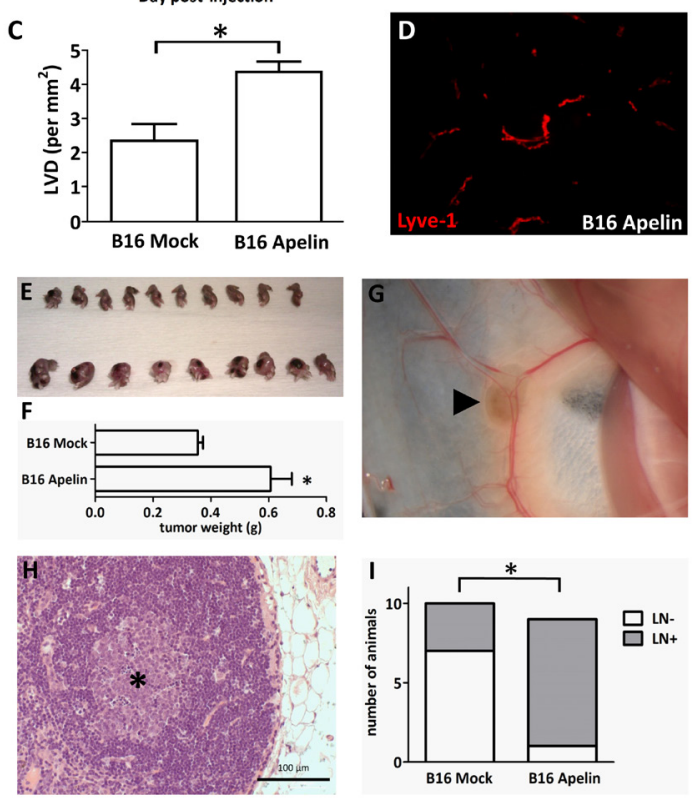

Figure 6: In vivo growth of apelin expressing tumor cells. (A), Overexpression of apelin through genetic manipulation significantly stimulated the in vivo growth of murine B16 melanoma cells in C57BL/6 mice. Growth curves of control vector (B16 Mock)- and apelin-transfected (B16 Apelin) cells. $\bullet$ and $\mathbf{\square}$, means for 10 mice per group; bars, SD; *, $\mathrm{P}<0.05$, versus control. (B-D), Apelin overexpression increases tumor-induced lymphangiogenesis in vivo. Frozen sections of 18-day-old control (B) and apelin-overexpressing (D) tumors were stained for the LEC marker LYVE-1 (red). Magnification, x200 (B, D). (C), LVDs (mean lymph vessel counts per square millimeter) of 18-day-old apelin-overexpressing or of control B16 tumors. Columns, means for ten mice per group; bars, SD; *, $\mathrm{P}<0.05$ vs. control. (E-F), Apelin-overexpressing B16 cells also developed significantly larger tumors (lower row) when injected into the footpads of mice $(*, P<0.05)$. Ipsilateral inguinal LN regions were carefully examined under a stereomicroscope, photographed and removed (G) (arrowhead shows pigmented B16 cells in the LN). (H), Histological demonstration of LN metastasis of B16 melanoma. Asterisk shows the colony of metastatic B16 cells in a LN. (I), The percentages of metastatic ipsilateral inguinal LNs (as evaluated by stereomicroscopy and histology) were $89 \%$ and $30 \%$ in the B16-Apelin and B16-Mock groups, respectively $(*, \mathrm{P}<0.05)$. 
or with an empty plasmid vector (B16-mock) [29]. In the first set of animal experiments, we implanted tumor cells subcutaneously into the upper back of mice and assessed tumor volumes (Fig. 6. A) and intratumoral LVDs (Fig. 6. B-D). Importantly, morphometrical analysis using LYVE1 as a LEC marker (Fig. 6. B, D) revealed significantly higher LVDs present in the apelin-overexpressing tumors as compared with controls $(\mathrm{P}<0.05$; Fig. 6. C). In addition, as expected [29], tumor growth was significantly accelerated in mice injected with B16-apelin cells compared with mice carrying B16-mock tumors (Fig. 6. A).

Stimulation of intratumoral lymph capillary formation by apelin raised the possibility that this peptide might also enhance lymphatic metastasis. Thus, to further explore the role of apelin in lymphatic tumor progression, B16-apelin and B16-mock cells were also injected into the right hind footpads of mice in groups of ten animals per cell line. Two weeks after implantation, B16 tumors increased rapidly in size. Three weeks after injection, when the primary tumors were $5-9 \mathrm{~mm}$ in diameter (Fig. 6. E-F), the animals were anaesthetized, and the ipsilateral inguinal LN regions were carefully examined under a stereomicroscope. In case of each animal, the LNs as well as the surrounding fat tissues were then removed and embedded into paraffin for serial sectioning. 8 of 9 (one mouse died at day 2 after tumor implantation in this group) animals (89\%) in the B16-apelin group showed metastases by stereomicroscopic inspection (Fig. 6. G; metastatic LN from one representative mouse) and histology (Fig. 6. H). By contrast, only 3 of 10 animals (30\%) in the B16-mock group had LN metastases (Fig. 6. I; $\mathrm{P}<0.05$ ).

\section{DISCUSSION}

Major progress has been made over the past two decades in understanding the molecular regulation of lymph vessel formation. Recent studies on lymphangiogenesis have been focused on two molecules of the VEGF (vascular endothelial growth factor) family, VEGF-C and -D, which are the ligands for VEGFR-3 (VEGF receptor-3) [1, 3]. Although various therapeutic drugs have already been generated to target this molecular machinery, recent results suggest that lymphangiogenesis is a complex process which is regulated by additional signaling pathways $[3,30]$. Further efforts are, therefore, needed for the better understanding of lymphatic biology to identify novel lymphangiogenic molecules and to develop more effective treatments for lymphangiogenesisrelated diseases, including cancer. In the present study, we provide evidence that apelin acts both in vitro and in vivo as a lymphangiogenic factor and, accordingly, that it has an important role in LN metastasis.

First, we showed that LECs express APJ in vitro and that apelin acts through APJ to increase Erk and Akt phosphorylation. In a recent study investigating the functions of APJ signaling during lymphatic development in zebrafish embryogenesis, Kim et al. also found that the knockdown of APJ in LECs selectively reduced the basal levels of phospho-Akt, but without affecting the phosphorylation status of Erk [19]. These authors, however, investigated the impact of APJ signaling abrogation on the basal phospho-Erk levels of LECs in the presence of FBS (fetal bovine serum), while we analyzed the effect of apelin stimulation in serum-deprived LECs, which raises the possibility that the lack of decrease in Erk phosphorylation in their study was due to their different experimental approach. The results presented in the current paper are indeed consistent with our group's earlier studies reporting that apelin induces the activation of both the PI3K-Akt and the Erk intracellular cascades in HUVECs (with endogenous APJ expression) and also in Chinese hamster ovary cells that have been stably transfected with APJ [8, 20]. Of note, both Akt [22, 24] and Erk [31-33] have also been shown to be downstream effectors of APJ signaling in various additional cell types including osteoblasts, vascular smooth muscle cells, embryonic kidney cells, neurons and cardiomyocytes (reviewed in ref. [34]). Additionally, VEGF-C/VEGFR3 in LECs are also known to activate Akt and Erk signaling and, moreover, both molecular pathways have been implicated in both developmental and pathological lymphangiogenesis $[35,36]$. Therefore, further studies are required to better understand the complex crosstalk among these intracellular cascades during lymphangiogenesis.

APJ activation has been demonstrated to promote cell migration in a variety of cell types including retinal endothelial [9], Müller glial [37], oral squamous cell carcinoma [16] and mesendodermal [38] cells. In line with these studies, we also found a significant pro-migratory effect of apelin on LECs. Of note, our results gained by using long-term time-lapse videomicroscopy in 2D cell cultures are also in accordance with the findings of the recent study of Sawane et al. [18], in which they used a transwell migration assay to evaluate the migratory response of LECs to apelin. It is also important to mention that the pro-migratory effect of apelin was reduced in a dose-dependent manner by the addition of F13A (an apelin mutant that has been considered as an APJ antagonist [14, 37, 39]). Our current findings, thus, suggest that F13A acts as a competitive antagonist on LECs when apelin-13 is present.

EC spheroids are increasingly used for evaluating the pro- and anti-angiogenic potential of various factors and drugs [40]. Furthermore, it has been demonstrated that endothelial cells retain differentiated phenotypic properties in 3D spheroids while they tend to lose them in monolayer cultures [26]. Accordingly, we also evaluated the effects of apelin on the formation of LEC spheroids, and found that although it did not have an effect on spheroid sizes, it increased significantly their numbers. This observation, taken together with our further in vitro finding that apelin 
stimulates the capillary-like tube formation of LECs, suggests an important role for apelin in the development of patent lymph capillaries via promoting a pro-adhesive state in the lymphatic endothelium. This assumption is also supported by recent studies reporting that apelin induces the endothelial expression of various intercellular adhesion molecules $[10,41,42]$ and, accordingly, that it enhances the integrity of both blood and lymph capillaries $[18,43]$.

The apelinergic system is expressed in a diverse range of tissues with particular predominance in the blood vasculature $[34,44]$. Accordingly, apelin has been shown to stimulate blood vascular EC growth in various in vitro $[8,9]$ and in vivo $[6,9]$ angiogenesis systems, including a mouse model of cancer [11]. Earlier studies have also provided evidence for apelin expression in human glioblastoma [12] and breast cancer [13]. We demonstrated recently a direct association of apelin expression with angiogenesis and clinical outcome in human non-small cell lung cancer [15] and, moreover, showed that autocrine apelin/APJ signaling participates in human colorectal cancer growth [14]. Another group demonstrated the prognostic significance of apelin expression in oral squamous cell carcinoma as well [16]. Most recently, circulating apelin levels were reported to be significantly increased in cancer patients (vs. healthy controls) and, moreover, to correlate with disease stage and prognosis [17]. Nevertheless, although the role of the apelinergic system during tumor progression and blood vessel formation has been an emerging focus of cancer and vascular research in recent years, whether and how apelin/APJ signaling contributes to tumorinduced lymphangiogenesis and lymphatic metastasis remains unclear. Thus, given the biological and clinical significance of apelin in the progression of human cancers, we hypothesized that it might enhance tumor progression by exerting a stimulatory effect on lymph vessel formation as well. Investigating the lymphangiogenic activity of apelin in the in vivo Matrigel plug model, we found that apelin-containing Matrigel plugs had significantly higher lymph vessel counts than those with PBS alone. This effect of apelin was comparable to that of bFGF which is known as a potent lymphangiogenic factor [45]. More importantly, apelin overexpression of murine B16 melanoma cells significantly increased the burden and intratumoral lymphangiogenesis of tumors growing subcutaneously in mice. These data were corroborated by the observation of enhanced LN metastasis in mice carrying apelin-transfected primary tumors. Two recent studies from Sawane et al. reported that apelin decreases UVB-induced lymphatic capillary hyperpermeability [18] and stabilizes lymph capillary walls in a high-fat diet mouse model [43]. These data further support our results and, taken together with our findings, suggest that the apelinergic system regulates the adult lymphatic endothelium under both physiological and pathological conditions. The very recent paper of Kim et al. about the essential role of the apelin/APJ pathway during embryonic lymphatic development [19] puts this idea into an even broader perspective implying that the effects of apelin/APJ signaling on LECs are conserved from fishes to mammals and throughout embryonic development to adulthood.

In conclusion, our study identifies apelin as a novel tumor lymphangiogenic factor that enhances LN metastasis. Accordingly, and based also on our group's previous studies, the apelinergic system stimulates tumor progression through the following mechanisms: 1./ promotion of blood capillary formation [11, 15], 2./ autocrine growth stimulation of malignant cells [14], and 3./ enhancement of intratumoral lymphangiogenesis and LN metastasis. Development of potential future therapies targeting this complex pro-tumor function of the apelin/ APJ pathway is thus especially intriguing as it might offer benefits to patients with malignant diseases.

\section{MATERIALS AND METHODS}

\section{Cells}

The B16 mouse melanoma cells stably transfected with murine apelin (B16-apelin) or with the empty plasmid vector (B16-mock) have been described in ref. [29]. HUVECs were purchased from Lonza (Walkersville, USA) and cultured in the manufacturer's recommended medium $\left(\mathrm{EGM}^{\circledR}-2\right.$ BulletKit $^{\circledR}$, Lonza) at $37^{\circ} \mathrm{C}$ in a humified incubator with $5 \% \mathrm{CO}_{2}$. Telomerase reverse transcriptase (TERT)-transduced LECs (isolated from human foreskins, and generated and characterized as described previously $[46,47])$, were grown in the same environment but in the EGM ${ }^{\circledR}-2-\mathrm{MV}$ BulletKit ${ }^{\circledR}$ (Lonza) medium.

\section{RNA isolation and real-time PCR}

Total RNA was extracted from HUVECs and LECs using TRIzol $\AA$ Reagent $\AA$ (Invitrogen) and purified with DNAfree DNase kit (Applied Biosystems, California, USA) according to the manufacturer's protocol. $2 \mu \mathrm{g}$ of total RNA from each sample were reverse transcribed using High-Capacity cDNA Reverse Transcription kit (Applied Biosystems) according to the manufacturer's protocol. Quantitative real-time PCR was performed using the cDNA as template with TaqMan Universal PCR Master mix (Applied Biosystems) and TaqMan premade gene expression assays (Applied Biosystems) to amplify APJ (Hs00270873_s1). All reactions were conducted as follows: $50^{\circ} \mathrm{C}$ for $2 \mathrm{~min}$ and 40 cycles of $10 \mathrm{~s}$ at $95^{\circ} \mathrm{C}$ and $1 \mathrm{~min}$ at $60^{\circ} \mathrm{C}$ in an Applied Biosystems 7500 Real-time PCR System. All samples were assayed in triplicate and control water samples were included in each experiment. 
The endogenous expression reference was the $\beta$-actin gene (Hs03023880_g1).

\section{APJ receptor protein expression in HUVECs and human LECs}

For immuncytochemical stainings, HUVECs and human LECs $\left(10^{5}\right.$ cells/well of a 24 -well plate) were plated on fibronectin-coated coverslips. After 16 hours, cells were fixed in 4\% PFA (10 min) and permeabilized with $0.1 \%$ Triton-X 100 solution (Sigma-Aldrich Co.) for 1 minute. Then the cells were incubated with a mouse antihuman APJ antibody (1:20; R\&D Systems, Minneapolis, USA). Biotinylated anti-mouse IgG (Vector Laboratories, California, USA) served as the secondary antibody and was detected by fluorescein streptavidin (Jackson Immunoresearch, West Grove, USA). For the negative control, the primary antibody was omitted and only the secondary antibody was applied. Finally, counterstaining was performed using propidium-iodide (PI; Partec, Görlitz, Germany). All samples were viewed by confocal laser scanning microscopy using the LSM 700 system (Carl Zeiss AG, Jena, Germany).

\section{In vitro Cell Proliferation Studies}

Cell proliferation was examined using the 5-bromo2'-deoxyuridine (BrdU; Sigma-Aldrich Co.) incorporation assay. $4 \times 10^{3}$ human LECs per well were plated in a flatbottomed 96-well plate in serum-free medium, and were treated with apelin-13 (Phoenix Pharmaceuticals, Karlsruhe, Germany) at $0.5,1,10$ and $100 \mathrm{nM}$ concentrations. After $96 \mathrm{~h}, 2 \mathrm{mg} / \mathrm{ml} \mathrm{BrdU}$ was added into the medium and the cells were incubated for an additional 2 hours at $37^{\circ} \mathrm{C}$. BrdU-positive cells were labeled with anti-BrdU antibody (Becton-Dickinson, New Jersey, USA). Biotinylated anti-mouse IgG (Vector Laboratories) served as the secondary antibody and was detected by fluorescein streptavidin (Jackson Immunoresearch). Finally, all nuclei were stained with PI (Partec). Three random fields from 3 wells were photographed and the number of BrdU-positive and PI-positive cells in each field counted. The percentage of the BrdU-positive cells was calculated and averaged.

\section{LEC spheroid growth assay}

To establish spheroid cultures from LECs, $3 \times 10^{4}$ cells were seeded in triplicate in DMEM/Ham's F-12 medium (PAA Laboratories, Pasching, Austria) supplemented with $20 \mathrm{ng} / \mathrm{ml}$ bFGF; (Eubio, Wien, Austria), $20 \mathrm{ng} / \mathrm{ml}$ epidermal growth factor (EGF; Sigma) and 2\% B27 supplement (PAA Laboratories) in ultra low attachment 24-well plates (Corning Inc., New York, USA).
Apelin-13 was added to the cells at $10 \mathrm{nM}$ or $1 \mu \mathrm{M}$ at the time of seeding. 96 hours after plating all spheroids in each well were photographed. In order to exclude small cell clumps from the analysis, only the spheres with a diameter over $100 \mu \mathrm{m}$ were counted and their diameter measured on the digital photographs using Image-J software.

\section{Migration assay}

The motility responses of human LECs to apelin were analysed by long-term time-lapse videomicroscopy in 2D cell cultures as described recently [25]. Briefly, LECs were plated in a 24-well plates (Corning Inc.) in a serum-free EBM-2 medium. The medium was changed to $\mathrm{CO}_{2}$-independent medium (Gibco, Paisley, UK) after the overnight cell attachment and apelin-13 or apelin13(F13A) (an APJ antagonist) [14, 37, 39] was added into the medium at 50 or $100 \mathrm{nM}$ concentrations. Cells were kept in a custom designed incubator built around an inverted phase-contrast microscope (World Precision Instruments, Florida, USA) at $37^{\circ} \mathrm{C}$ and room ambient atmosphere. Images of 3 neighbouring microscopic fields were taken in every $5 \mathrm{~min}$ for 1 day before and 2 days after the treatment. For migration data, pictures were analysed individually with a cell-tracking program enabling manual marking of individual cells and recording their position parameters. The parameter migrated distance is calculated by averaging for each cell the displacement for the first 24 hour interval after treatment, in two independent experiments and 4 microscopic fields.

\section{Tube formation assay}

Liquefied $10 \mathrm{mg} / \mathrm{ml}$ Matrigel Basement Membrane Matrix (0.289 ml/well; BD Biosciences, New Jersey, USA) was used to coat wells of a 24-well plate and then allowed to polymerize at $37^{\circ} \mathrm{C}$ for a minimum of $30 \mathrm{~min} .10^{5}$ cells per well were seeded on basal membrane extract in serumfree, EBM-2 medium (Lonza). The cells were treated with $1 \mu \mathrm{M}$ apelin-13 (Phoenix) or $20 \mathrm{ng} / \mathrm{ml} \mathrm{FGF}$ (Eubio) and incubated $16-18$ hours at $37^{\circ} \mathrm{C}$. Capillary-like structures within the Matrigel layer were then photographed and analyzed using Image J morphometry software.

\section{Apoptosis assay}

$5 \times 10^{4}$ cells per well were cultured in a 24 -well plate. After 24 hours, the cells were treated with $1 \mu \mathrm{M}$ apelin-13. Apoptosis of cells was induced with an UV dose of $200 \mathrm{~mW} / \mathrm{cm} 2$ for 10 seconds. After 48 hours incubation, cells were fixed in 4\% PFA for $10 \mathrm{~min}$. Apoptosis assay was carried out using the "In Situ Cell Death Detection" kit (Roche, Mannheim, Germany) and the protocol recommended by the manufacturer. Finally, cells were 
coverslipped in Vectashield mounting medium containing DAPI (Vector Laboratories). Five random fields per slide were photographed and the percentage of TUNEL-positive cells among total cells was calculated and averaged.

\section{Western Blot Analysis}

LECs exposed to $1 \mu \mathrm{M}$ apelin in serum-free medium (EBM-2, Lonza) for different time durations were lysed in RIPA-buffer (Fischer Scientific, Loughborough, UK), centrifuged, and protein was denatured before loading. Each sample was subjected to $12 \%$ sodium dodecyl sulfate (SDS)-polyacrylamide gel electrophoresis. The proteins were electro-transferred onto nitrocellulose membranes and immunodetected with antibodies against AktpAkt, Erk1/2-pErk1/2, S6-pS6 (all from Cell Signaling Technologies, Massachusetts, USA) and $\beta$-actin (Sigma). Blots were then incubated with appropriate HRP-labeled secondary antibodies (Thermo Fisher Scientific, Rockford, IL, USA) and signals were detected by using the ECL system (GE Healthcare, Dassel, Germany). Activation of signaling was quantified as the ratio of phosphorylated and total protein densitometry measurements.

\section{Matrigel plug assay}

Groups of three C57BL/6 mice were injected subcutaneously with $0.25 \mathrm{ml}$ Matrigel (BD Biosciences) containing PBS, $0.25 \mu \mathrm{g}$ bFGF (Eubio), or $0.25 \mu \mathrm{g}$ apelin (Phoenix Pharmaceuticals). One week after implantation, the plug was removed, and cryosections $(5 \mu \mathrm{m})$ were prepared for immunohistochemistry. After labeling with rabbit anti-mouse LYVE-1 and rhodamine-conjugated goat anti-rabbit IgG (purchased from ReliaTech, Braunschweig, Germany and Jackson ImmunoResearch Inc., West Grove, PA, respectively), sections were examined using a Nikon Eclipse 80i microscope and digital images were captured using a SPOT digital camera (Diagnostic Instruments, Sterling Heights, MI). Quantitative analyses of the LYVE-1-positive lymphatic vessels in the Matrigels were performed using ImageJ software.

\section{In vivo tumor studies}

Growth of the apelin-transfected B16 mouse melanoma cells was compared with that of control vector expressing cells in allograft tumors formed in 9-week-old female BDF1 ((C57BL/6 x DBA/2)F1) mice. According to the institutional animal welfare guidelines, all mice were maintained on a daily 12-h light/12-h dark cycle and were housed under pathogen-free conditions in microisolator cages with laboratory chow and water ad libitum. B16-apelin and B16-mock cells were grown to $80 \%$ confluence, harvested by trypsinization and washed twice. Tumor allografts were established by injecting mice subcutaneously with $1 \times 10^{6}$ B16-apelin or B16-mock cells under anesthesia with a combination of tiletamine hypochloride, zolazepam hypochloride, xylazin and butorphanol. Tumor size was measured every two days with a caliper and expressed in $\mathrm{mm}^{3}$ by the formula for the volume of a prolate ellipsoid (length $\mathrm{x}$ width ${ }^{2} \pi / 6$ ), as described previously $[15,48]$. Tumors were removed from mice after 18 days of growth and were fresh-frozen in liquid nitrogen for further analysis. Lymph vessel densities (LVDs) were determined by labeling of lymph capillaries with rabbit anti-mouse LYVE-1 (ReliaTech) and rhodamine-conjugated goat anti-rabbit IgG (Jackson ImmunoResearch). Three sections per tumor were analyzed using a Nikon Eclipse 80i microscope. Digital images were captured and analyzed with a SPOT digital camera (Diagnostic Instruments) and the ImageJ software, respectively and as described previously $[49,50]$.

To study the LN metastatic potential of B16apelin primary tumors, in another set of in vivo tumor experiments, groups of ten mice were injected with B16apelin or B16-mock cells $\left(1 \times 10^{6}\right.$ cells per animal) in a total volume of $100 \mu \mathrm{l}$ into the right hind footpad. Animals were euthanized and autopsied at 3 weeks postinoculation when the primary tumors reached $5-10 \mathrm{~mm}$ in diameter. Metastatic involvement of the ipsilateral inguinal sentinel LNs was evaluated with a stereomicroscope (Alpha, Woking, UK). The right-side inguinal LN of each animal were then collected, embedded in paraffin, serial sectioned, stained with haematoxylin and eosin and evaluated by a pathologist (BD).

\section{Statistical analysis}

Continuous variables were compared with Student's t-test if the sample distribution was normal or with MannWhitney $U$ test if the sample distribution was asymmetric. Categorical data were compared using Fisher's exact probability and $\chi^{2}$ tests. Differences were determined to be significant if $\mathrm{p}<0.05$. All statistical analyses were done using GraphPad Prism 5.0 (GraphPad Software, Inc.; La Jolla, CA) software.

\section{ACKNOWLEDGEMENTS}

The authors were supported by KTIA AIK 121-2013-0041 (BD, AR, TG, VL, SP, FRV); TÁMOP 424A/1-11-1-2012-0001 (BD); OTKA K109626, OTKA K108465 (BD, BH), OTKA MOB80325 (BH); OTKA K84173 (JT); EUREKA_HU_12-1-2012-0057 (BD); ÖNB Jubiläumsfondsprojekt Nr. 14043 (BD, VL, AR) and Nr. 14574 (MAH); Ligue Régionale contre le Cancer (BM) and the Vienna Fund for Innovative Interdisciplinary Cancer Research (BD, VL). 


\section{Conflict of interest statement}

The authors declare no potential conflicts of interest.

\section{REFERENCES}

1. Karpanen T and Alitalo K. Molecular biology and pathology of lymphangiogenesis. Annu Rev Pathol. 2008; 3:367-397.

2. Saito Y, Nakagami H, Kaneda $Y$ and Morishita R. Lymphedema and therapeutic lymphangiogenesis. Biomed Res Int. 2013; 2013:804675.

3. Stacker SA, Williams SP, Karnezis T, Shayan R, Fox SB and Achen MG. Lymphangiogenesis and lymphatic vessel remodelling in cancer. Nat Rev Cancer. 2014; 14(3):159172.

4. Pitkin SL, Maguire JJ, Bonner TI and Davenport AP. International Union of Basic and Clinical Pharmacology. LXXIV. Apelin receptor nomenclature, distribution, pharmacology, and function. Pharmacol Rev. 2010; 62(3):331-342.

5. Devic E, Rizzoti K, Bodin S, Knibiehler B and Audigier Y. Amino acid sequence and embryonic expression of $\mathrm{msr} /$ apj, the mouse homolog of Xenopus X-msr and human APJ. Mech Dev. 1999; 84(1-2):199-203.

6. Cox CM, D'Agostino SL, Miller MK, Heimark RL and Krieg PA. Apelin, the ligand for the endothelial G-proteincoupled receptor, APJ, is a potent angiogenic factor required for normal vascular development of the frog embryo. Dev Biol. 2006; 296(1):177-189.

7. Kleinz MJ, Skepper JN and Davenport AP. Immunocytochemical localisation of the apelin receptor, APJ, to human cardiomyocytes, vascular smooth muscle and endothelial cells. Regul Pept. 2005; 126(3):233-240.

8. Masri B, Morin N, Cornu M, Knibiehler B and Audigier Y. Apelin (65-77) activates p70 S6 kinase and is mitogenic for umbilical endothelial cells. FASEB J. 2004; 18(15):19091911.

9. Kasai A, Shintani N, Oda M, Kakuda M, Hashimoto H, Matsuda T, Hinuma S and Baba A. Apelin is a novel angiogenic factor in retinal endothelial cells. Biochem Biophys Res Commun. 2004; 325(2):395-400.

10. Kidoya H, Kunii N, Naito H, Muramatsu F, Okamoto Y, Nakayama $\mathrm{T}$ and Takakura N. The apelin/APJ system induces maturation of the tumor vasculature and improves the efficiency of immune therapy. Oncogene. 2012; 31(27):3254-3264.

11. Sorli SC, Le Gonidec S, Knibiehler B and Audigier Y. Apelin is a potent activator of tumour neoangiogenesis. Oncogene. 2007; 26(55):7692-7699.

12. Kalin RE, Kretz MP, Meyer AM, Kispert A, Heppner FL and Brandli AW. Paracrine and autocrine mechanisms of apelin signaling govern embryonic and tumor angiogenesis. Dev Biol. 2007; 305(2):599-614.
13. Wang Z, Greeley GH, Jr. and Qiu S. Immunohistochemical localization of apelin in human normal breast and breast carcinoma. J Mol Histol. 2008; 39(1):121-124.

14. Picault FX, Chaves-Almagro C, Projetti F, Prats H, Masri $\mathrm{B}$ and Audigier Y. Tumour co-expression of apelin and its receptor is the basis of an autocrine loop involved in the growth of colon adenocarcinomas. Eur J Cancer. 2014; 50(3):663-674.

15. Berta J, Kenessey I, Dobos J, Tovari J, Klepetko W, Jan Ankersmit H, Hegedus B, Renyi-Vamos F, Varga J, Lorincz Z, Paku S, Ostoros G, Rozsas A, Timar J and Dome B. Apelin expression in human non-small cell lung cancer: role in angiogenesis and prognosis. J Thorac Oncol. 2010; 5(8):1120-1129.

16. Heo K, Kim YH, Sung HJ, Li HY, Yoo CW, Kim JY, Park JY, Lee UL, Nam BH, Kim EO, Kim SY, Lee SH, Park JB and Choi SW. Hypoxia-induced up-regulation of apelin is associated with a poor prognosis in oral squamous cell carcinoma patients. Oral Oncol. 2012; 48(6):500-506.

17. Lacquaniti A, Altavilla G, Picone A, Donato V, Chirico V, Mondello P, Aloisi C, Marabello G, Loddo S, Buemi A, Lorenzano G and Buemi M. Apelin beyond kidney failure and hyponatremia: a useful biomarker for cancer disease progression evaluation. Clin Exp Med. 2014.

18. Sawane M, Kidoya H, Muramatsu F, Takakura N and Kajiya K. Apelin attenuates UVB-induced edema and inflammation by promoting vessel function. Am J Pathol. 2011; 179(6):2691-2697.

19. Kim JD, Kang Y, Kim J, Papangeli I, Kang H, Wu J, Park H, Nadelmann E, Rockson SG, Chun HJ and Jin SW. Essential role of Apelin signaling during lymphatic development in zebrafish. Arterioscler Thromb Vasc Biol. 2014; 34(2):338-345.

20. Masri B, Lahlou H, Mazarguil H, Knibiehler B and Audigier Y. Apelin (65-77) activates extracellular signalregulated kinases via a PTX-sensitive $\mathrm{G}$ protein. Biochem Biophys Res Commun. 2002; 290(1):539-545.

21. Wang XL, Tao Y, Lu Q and Jiang YR. Apelin supports primary rat retinal Muller cells under chemical hypoxia and glucose deprivation. Peptides. 2012; 33(2):298-306.

22. Tang SY, Xie H, Yuan LQ, Luo XH, Huang J, Cui RR, Zhou HD, Wu XP and Liao EY. Apelin stimulates proliferation and suppresses apoptosis of mouse osteoblastic cell line MC3T3-E1 via JNK and PI3-K/Akt signaling pathways. Peptides. 2007; 28(3):708-718.

23. Zeng XJ, Yu SP, Zhang L and Wei L. Neuroprotective effect of the endogenous neural peptide apelin in cultured mouse cortical neurons. Exp Cell Res. 2010; 316(11):17731783.

24. Cui RR, Mao DA, Yi L, Wang C, Zhang XX, Xie H, Wu XP, Liao XB, Zhou H, Meng JC, Yuan LQ and Liao EY. Apelin suppresses apoptosis of human vascular smooth muscle cells via APJ/PI3-K/Akt signaling pathways. Amino Acids. 2010; 39(5):1193-1200. 
25. Garay T, Juhasz E, Molnar E, Eisenbauer M, Czirok A, Dekan B, Laszlo V, Hoda MA, Dome B, Timar J, Klepetko W, Berger W and Hegedus B. Cell migration or cytokinesis and proliferation?--revisiting the „go or grow” hypothesis in cancer cells in vitro. Exp Cell Res. 2013; 319(20):30943103.

26. Korff $\mathrm{T}$ and Augustin HG. Integration of endothelial cells in multicellular spheroids prevents apoptosis and induces differentiation. J Cell Biol. 1998; 143(5):1341-1352.

27. Takakura $\mathrm{N}$ and Kidoya H. Maturation of blood vessels by haematopoietic stem cells and progenitor cells: involvement of apelin/APJ and angiopoietin/Tie2 interactions in vessel caliber size regulation. Thromb Haemost. 2009; 101(6):9991005.

28. Staton CA, Reed MW and Brown NJ. A critical analysis of current in vitro and in vivo angiogenesis assays. Int J Exp Pathol. 2009; 90(3):195-221.

29. Sorli SC, van den Berghe L, Masri B, Knibiehler B and Audigier $Y$. Therapeutic potential of interfering with apelin signalling. Drug Discov Today. 2006; 11(23-24):11001106.

30. Duong T, Koopman $\mathrm{P}$ and Francois $\mathrm{M}$. Tumor lymphangiogenesis as a potential therapeutic target. J Oncol. 2012; 2012:204946.

31. Bai B, Tang J, Liu H, Chen J, Li Y and Song W. Apelin-13 induces ERK1/2 but not p38 MAPK activation through coupling of the human apelin receptor to the Gi2 pathway. Acta Biochim Biophys Sin (Shanghai). 2008; 40(4):311318.

32. O’Donnell LA, Agrawal A, Sabnekar P, Dichter MA, Lynch DR and Kolson DL. Apelin, an endogenous neuronal peptide, protects hippocampal neurons against excitotoxic injury. J Neurochem. 2007; 102(6):1905-1917.

33. Simpkin JC, Yellon DM, Davidson SM, Lim SY, Wynne AM and Smith CC. Apelin-13 and apelin-36 exhibit direct cardioprotective activity against ischemia-reperfusion injury. Basic Res Cardiol. 2007; 102(6):518-528.

34. O'Carroll AM, Lolait SJ, Harris LE and Pope GR. The apelin receptor APJ: journey from an orphan to a multifaceted regulator of homeostasis. J Endocrinol. 2013; 219(1):R13-35.

35. Zhou F, Chang Z, Zhang L, Hong YK, Shen B, Wang B, Zhang F, Lu G, Tvorogov D, Alitalo K, Hemmings $\mathrm{BA}$, Yang $\mathrm{Z}$ and $\mathrm{He} \mathrm{Y}$. Akt/Protein kinase B is required for lymphatic network formation, remodeling, and valve development. Am J Pathol. 2010; 177(4):2124-2133.

36. Deng Y, Atri D, Eichmann A and Simons M. Endothelial ERK signaling controls lymphatic fate specification. J Clin Invest. 2013; 123(3):1202-1215.

37. Lu Q, Jiang YR, Qian J and Tao Y. Apelin-13 regulates proliferation, migration and survival of retinal Muller cells under hypoxia. Diabetes Res Clin Pract. 2013; 99(2):158167.

38. Pauli A, Norris ML, Valen E, Chew GL, Gagnon JA,
Zimmerman S, Mitchell A, Ma J, Dubrulle J, Reyon D, Tsai SQ, Joung JK, Saghatelian A and Schier AF. Toddler: an embryonic signal that promotes cell movement via Apelin receptors. Science. 2014; 343(6172):1248636.

39. Lee DK, Saldivia VR, Nguyen T, Cheng R, George SR and O'Dowd BF. Modification of the terminal residue of apelin-13 antagonizes its hypotensive action. Endocrinology. 2005; 146(1):231-236.

40. Hirschhaeuser F, Menne H, Dittfeld C, West J, MuellerKlieser W and Kunz-Schughart LA. Multicellular tumor spheroids: an underestimated tool is catching up again. $\mathrm{J}$ Biotechnol. 2010; 148(1):3-15.

41. Kidoya H, Ueno M, Yamada Y, Mochizuki N, Nakata M, Yano T, Fujii R and Takakura N. Spatial and temporal role of the apelin/APJ system in the caliber size regulation of blood vessels during angiogenesis. EMBO J. 2008; 27(3):522-534.

42. Lu Y, Zhu X, Liang GX, Cui RR, Liu Y, Wu SS, Liang QH, Liu GY, Jiang Y, Liao XB, Xie H, Zhou HD, Wu XP, Yuan LQ and Liao EY. Apelin-APJ induces ICAM-1, VCAM-1 and MCP-1 expression via NF-kappaB/JNK signal pathway in human umbilical vein endothelial cells. Amino Acids. 2012; 43(5):2125-2136.

43. Sawane M, Kajiya K, Kidoya H, Takagi M, Muramatsu F and Takakura N. Apelin inhibits diet-induced obesity by enhancing lymphatic and blood vessel integrity. Diabetes. 2013; 62(6):1970-1980.

44. Medhurst AD, Jennings CA, Robbins MJ, Davis RP, Ellis C, Winborn KY, Lawrie KW, Hervieu G, Riley G, Bolaky JE, Herrity NC, Murdock P and Darker JG. Pharmacological and immunohistochemical characterization of the APJ receptor and its endogenous ligand apelin. J Neurochem. 2003; 84(5):1162-1172.

45. Chang LK, Garcia-Cardena G, Farnebo F, Fannon M, Chen EJ, Butterfield C, Moses MA, Mulligan RC, Folkman J and Kaipainen A. Dose-dependent response of FGF-2 for lymphangiogenesis. Proc Natl Acad Sci U S A. 2004; 101(32):11658-11663.

46. Groger M, Loewe R, Holnthoner W, Embacher R, Pillinger $\mathrm{M}$, Herron GS, Wolff $\mathrm{K}$ and Petzelbauer P. IL-3 induces expression of lymphatic markers Prox-1 and podoplanin in human endothelial cells. J Immunol. 2004; 173(12):71617169.

47. Bracher A, Cardona AS, Tauber S, Fink AM, Steiner A, Pehamberger H, Niederleithner H, Petzelbauer P, Groger $\mathrm{M}$ and Loewe R. Epidermal growth factor facilitates melanoma lymph node metastasis by influencing tumor lymphangiogenesis. J Invest Dermatol. 2013; 133(1):230238.

48. Rozsas A, Berta J, Rojko L, Horvath LZ, Keszthelyi M, Kenessey I, Laszlo V, Berger W, Grusch M, Hoda MA, Torok S, Klepetko W, Renyi-Vamos F, Hegedus B, Dome B and Tovari J. Erythropoietin receptor expression is a potential prognostic factor in human lung adenocarcinoma. PLoS One. 2013; 8(10):e77459. 
49. Dome B, Paku S, Somlai B and Timar J. Vascularization of cutaneous melanoma involves vessel co-option and has clinical significance. J Pathol. 2002; 197(3):355-362.

50. Paku S, Kopper L and Nagy P. Development of the vasculature in "pushing-type" liver metastases of an experimental colorectal cancer. Int J Cancer. 2005; 115(6):893-902. 